\title{
Unified Atomic Mass Unit
}

National Cancer Institute

\section{Source}

National Cancer Institute. Unified Atomic Mass Unit. NCI Thesaurus. Code C41127.

The non-SI unit of mass in terms of which the masses of individual atoms and molecules are expressed. Unified atomic mass unit is defined as one twelfth of the mass of an unbound atom of the carbon-12 nuclide, the predominant isotope of carbon, at rest and in its ground state. It is equal to $1.6606 \times 10(\mathrm{E}-27) \mathrm{kg}$ (approximately). 